\section{(2) OPEN ACCESS}

\title{
Association of social relationships with incident cardiovascular events and all-cause mortality
}

\author{
Janine Gronewold (1) , ${ }^{1}$ Rene Kropp, ${ }^{1}$ Nils Lehmann, ${ }^{2}$ Börge Schmidt, ${ }^{2}$ \\ Simone Weyers, ${ }^{3}$ Johanne Siegrist, ${ }^{3}$ Nico Dragano, ${ }^{3}$ Karl-Heinz Jöckel, ${ }^{2}$ Raimund Erbel, ${ }^{2}$ \\ Dirk M Hermann, ${ }^{1}$ on behalf of the Heinz Nixdorf Recall Study Investigative Group
}

\begin{abstract}
- Additional material is published online only. To view please visit the journal online (http://dx.doi.org/10.1136/ heartjnl-2019-316250).

${ }^{1}$ Department of Neurology, University Hospital Essen, Essen, Germany

Institute for Medical Informatics, Biometry and Epidemiology, University Duisburg-Essen, Essen, Germany ${ }^{3}$ Institute for Medical Sociology, Medical Faculty, HeinrichHeine-University Düsseldorf, Düsseldorf, Germany
\end{abstract}

\section{Correspondence to} Professor Dirk M Hermann, Neurology, University Hospital Essen, Essen, Germany; dirk.hermann@uk-essen.de

Received 15 November 2019 Revised 7 January 2020 Accepted 17 February 2020 Published Online First 12 March 2020
Check for updates

(C) Author(s) (or their employer(s)) 2020. Re-use permitted under CC BY-NC. No commercial re-use. See rights and permissions. Published by BMJ.

To cite: Gronewold J, Kropp R, Lehmann N, et al. Heart 2020;106:1317-1323.

\section{ABSTRACT}

Objective To examine how different aspects of social relationships are associated with incident cardiovascular events and all-cause mortality.

Methods In 4139 participants from the populationbased Heinz Nixdorf Recall study without previous cardiovascular disease (mean (SD) age 59.1 (7.7) years, $46.7 \% \mathrm{men})$, the association of self-reported instrumental, emotional and financial support and social integration at baseline with incident fatal and non-fatal cardiovascular events and all-cause mortality during 13.4-year follow-up was assessed in five different multivariable Cox proportional hazards regression models: minimally adjusted model (adjusting for age, sex, social integration or social support, respectively); biological model (minimally adjusted+systolic blood pressure, low-density and high-density lipoprotein cholesterol, glycated haemoglobin, body mass index, antihypertensive medication, lipid-lowering medication and antidiabetic medication); health behaviour model (minimally adjusted+alcohol consumption, smoking and physical activity); socioeconomic model (minimally adjusted+income, education and employment); and depression model (minimally adjusted+depression, antidepressants and anxiolytics).

Results 339 cardiovascular events and 530 deaths occurred during follow-up. Lack of financial support was associated with an increased cardiovascular event risk (minimally adjusted $\mathrm{HR}=1.30(95 \% \mathrm{Cl} 1.01$ to 1.67)). Lack of social integration (social isolation) was associated with increased mortality (minimally adjusted $H R=1.47(95 \%$ Cl 1.09 to 1.97)). Effect estimates did not decrease to a relevant extent in any regression model.

Conclusions Perceiving a lack of financial support is associated with a higher cardiovascular event incidence, and being socially isolated is associated with increased all-cause mortality. Future studies should investigate how persons with deficient social relationships could benefit from targeted interventions.

\section{INTRODUCTION}

Feeling lonely or being socially isolated is associated with a higher risk for cardiovascular events ${ }^{1}$ and all-cause mortality. ${ }^{2}$ There is both evidence that the association of social relationships with incident morbidity and mortality is to a large degree explained by traditional clinical risk factors ${ }^{3}$ and that social relationships influence incident morbidity and mortality independent from the modification of traditional clinical risk factors. ${ }^{4}$ Social relationships have already been shown to exert a relevant influence on mortality in epidemiological studies ${ }^{4}$ even though pathomechanisms remain unclear. ${ }^{5}$ Previous studies did not distinguish between the effects of qualitative aspects of social relationships (eg, being able to receive support from social relationships, representing social support) versus quantitative aspects (eg, number of persons to contact or frequency of contacts, representing social integration). ${ }^{1}$ While qualitative aspects of perceived social support in terms of instrumental, emotional and financial support were previously linked to mortality, ${ }^{6}$ a combined analysis of quantitative and qualitative aspects of social relationships with incident cardiovascular events and all-cause mortality has not yet been performed. To fill this gap of knowledge, we focused on the association of both qualitative aspects (perceived social support, classified by the types instrumental, emotional and financial) and quantitative aspects (social integration) of social relationships, with incident cardiovascular events and all-cause mortality in the prospective, population-based Heinz Nixdorf Recall study. Furthermore, we analysed to which extent these associations are explained by cardiovascular risk factors, socioeconomic factors, health behaviour or emotional factors.

\section{METHODS}

\section{Study participants}

Data were drawn from the Heinz Nixdorf Recall study, a prospective population-based study focusing on risk factors for cardiovascular disease and death. A random sample of men and women aged 45-75 years were enrolled via mandatory citizen registries in Essen, Bochum and Mülheim/Ruhr between December 2000 and August 2003 and received two follow-up examinations after 5 years and 10 years. The study design has been described in detail elsewhere. ${ }^{7}$ All participants gave written informed consent. The study was approved by the ethical committee of the University of Duisburg-Essen, Germany. From the total cohort of 4814 Heinz Nixdorf Recall study participants, 4316 were free of vascular disease (stroke, coronary heart disease and peripheral artery disease) at the baseline examination. Of those, 4139 participants (mean (SD) age 59.1 (7.7) years, $46.7 \%$ men) had complete information about social support and social integration, 
representing the sample for the following analyses. Patients or the public were not involved in the design, conduct, reporting or dissemination plans of our research.

\section{Social relationships}

Social support

We assessed three types of social support (instrumental, emotional and financial). Instrumental and emotional social support were measured with a German adaptation of the New Haven Established Populations for Epidemiologic Studies of the Elderly (New Haven EPESE) questionnaire in a computerassisted personal interview. ${ }^{8}$ This questionnaire first asks for the availability of someone to help in daily tasks like buying groceries, house cleaning, preparing meals (instrumental support) and someone to approach in case of emotional problems (emotional support). If support was available, participants were asked who actually provided support during the previous 12 months or whether support was not needed during that time. If participants reported persons who actually provided support, they were asked whether that support had been sufficient. For financial support, self-constructed questions analogue to the New Haven EPESE were used. Additionally, the participants could specify if they had refused offered financial support. Based on the combination of this information, four categories were defined: 'support not needed' (someone available but not needed), 'support adequate' (someone provided support, this support was sufficient), 'support inadequate' (someone provided support, this support was insufficient) and 'no support available' (no one available). Lack of instrumental or emotional support was defined as one of the latter two categories; for financial support, refusal of offered support was additionally classified as lack of support. ${ }^{9}$

\section{Social integration}

We assessed social integration with the social integration index developed by Berkman et al. ${ }^{10}$ The index includes three types of ties: (1) marital status/cohabitation, (2) contacts with close friends/family and (3) affiliation with voluntary associations. These types of ties each scored from 0 to 2, thus the index ranged from 0 to 6. Marital status/cohabitation was scored as 0 if the participant was single, divorced or widowed and 2 if the subject was married or living with a partner. Contacts with close friends and family was scored as 0 in case of $0-2$ contacts, 1 in case of $3-11$ contacts and 2 in case of $\geq 12$ contacts. Affiliation with voluntary associations was defined by membership in any of six types of political, religious, community, sports or professional organisations and scored as 0 in case of no membership, 1 in case of membership in one organisation, and two in case of membership in $\geq 2$ organisations. The social integration index was categorised into four levels: level I included persons who scored 0 or 1 , and levels II, III and IV included persons who scored 2-3, 4-5 and 6, respectively. We defined lack of social integration (social isolation) by level I.

\section{Confounding variables}

The measurement of confounding variables at the baseline examination is described in the online supplementary methods.

\section{Follow-up}

Participants were followed over a median of 13.4 (IQR 10.514.2) years for the endpoint of cardiovascular events (strokes, coronary events or independently coded causes of deaths according to diseases of the circulatory system; detailed study endpoint definitions are given in the online supplementary methods). The data set was closed for the purpose of this study in August 2017; the last participant follow-up was on 28 April 282017.

\section{Statistical analysis}

Continuous data are presented as mean (SD) for normally distributed or median (IQR) for non-normally distributed data; categorical data are shown as number (\%). Statistical comparisons between participants reporting a lack of social support/ social integration versus no lack of social support/social integration were done by independent two-sample t-test for normally distributed data, by Mann-Whitney tests for non-normally distributed data and by $\chi^{2}$ tests for categorical data. Multivariable Cox proportional hazards models were used to evaluate the relationship between lack of social support/social integration and risk of cardiovascular events as well as all-cause mortality. Analysis of Schoenfeld residuals and a Kolmogorov-type supremum test confirmed validity of the proportional hazards assumption. Incident non-fatal cardiovascular events after the baseline examination were allocated to the date of diagnosis, incident fatal cardiovascular events to the date of death according to the death certificate, participants not experiencing cardiovascular events during the follow-up were censored at the date of last contact when the person was still alive or date of death not caused by diseases of the circulatory system. Similar to a previous analysis by Hakulinen et al, ${ }^{3}$ who analysed the association of social isolation and feeling lonely with incident myocardial infarction and stroke, we used the following adjustment models:

- Minimally adjusted (adjusting for age, sex and social integration or social support, respectively).

- Biological factors (minimally adjusted+systolic blood pressure, low-density lipoprotein cholesterol, high-density lipoprotein cholesterol, glycated haemoglobin, body mass index, antihypertensive medication, lipid-lowering medication and antidiabetic medication).

- Health behaviour (minimally adjusted+alcohol consumption, current smoking and regular physical activity).

- Socioeconomic (minimally adjusted+income, education and employment).

- Depression (minimally adjusted+depression, antidepressants and anxiolytics).

Missing values were excluded listwise. All analyses were done with IBM SPSS Statistics V.21 for Windows.

\section{RESULTS}

\section{Baseline characteristics}

The descriptive statistics of the study cohort, also stratified by the perceived adequacy of the different types of social support and by social integration, are presented in table 1 .

Participants with lack of instrumental support were more likely to be female, unemployed, physically inactive, taking antidepressants, had higher depression scores, lower income, a lower level of education and drank less alcohol.

Participants who reported lack of emotional support were also more likely to be unemployed, taking antidepressants, had higher depression scores, a smaller social network, lower income and a lower level of education.

Participants with lack of financial support similar to participants with lack of instrumental and emotional support were more likely to be unemployed, physically inactive, had higher depression scores, lower income and a lower level of education. 


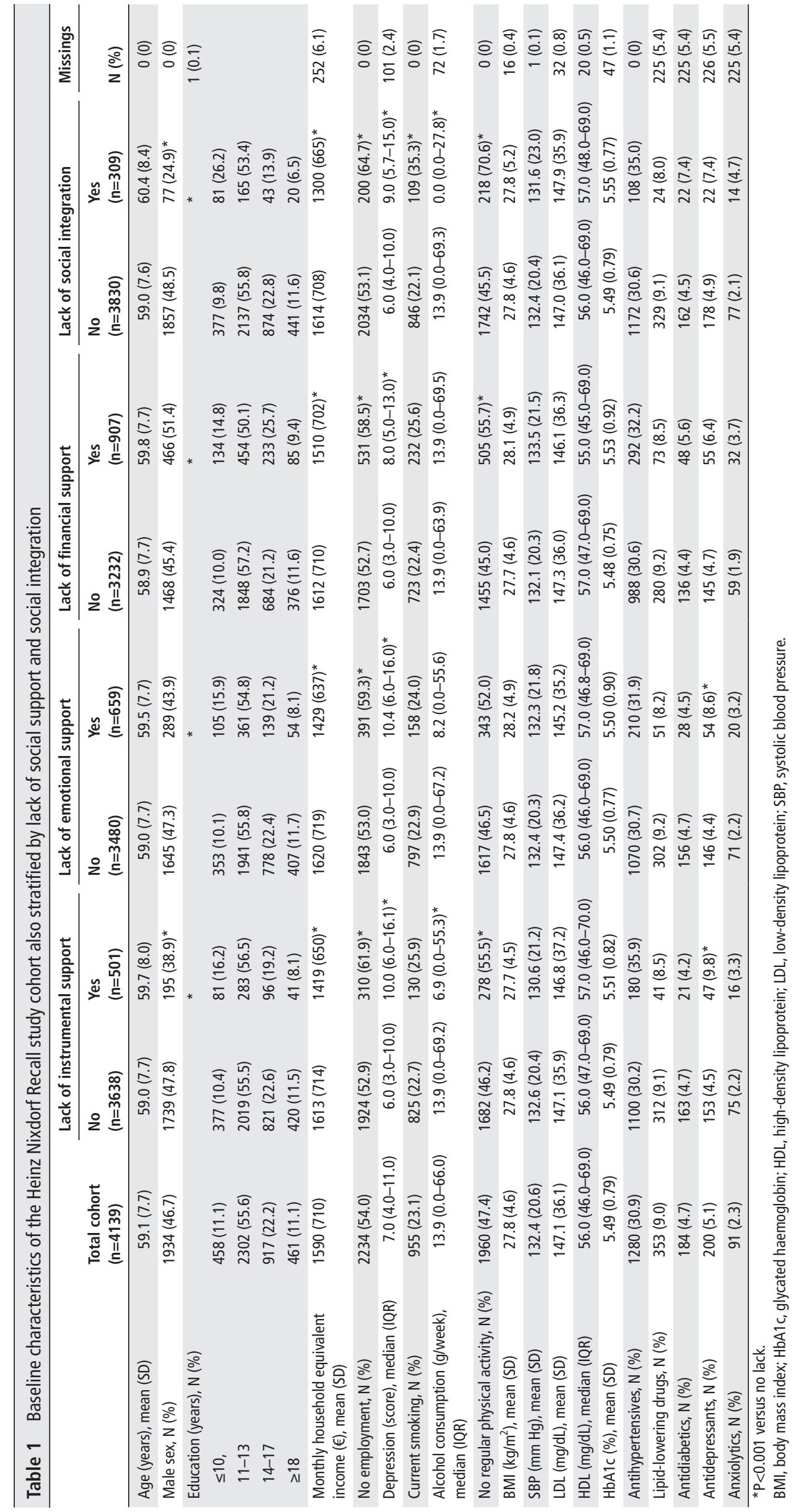




\section{Cardiovascular events}

Social support

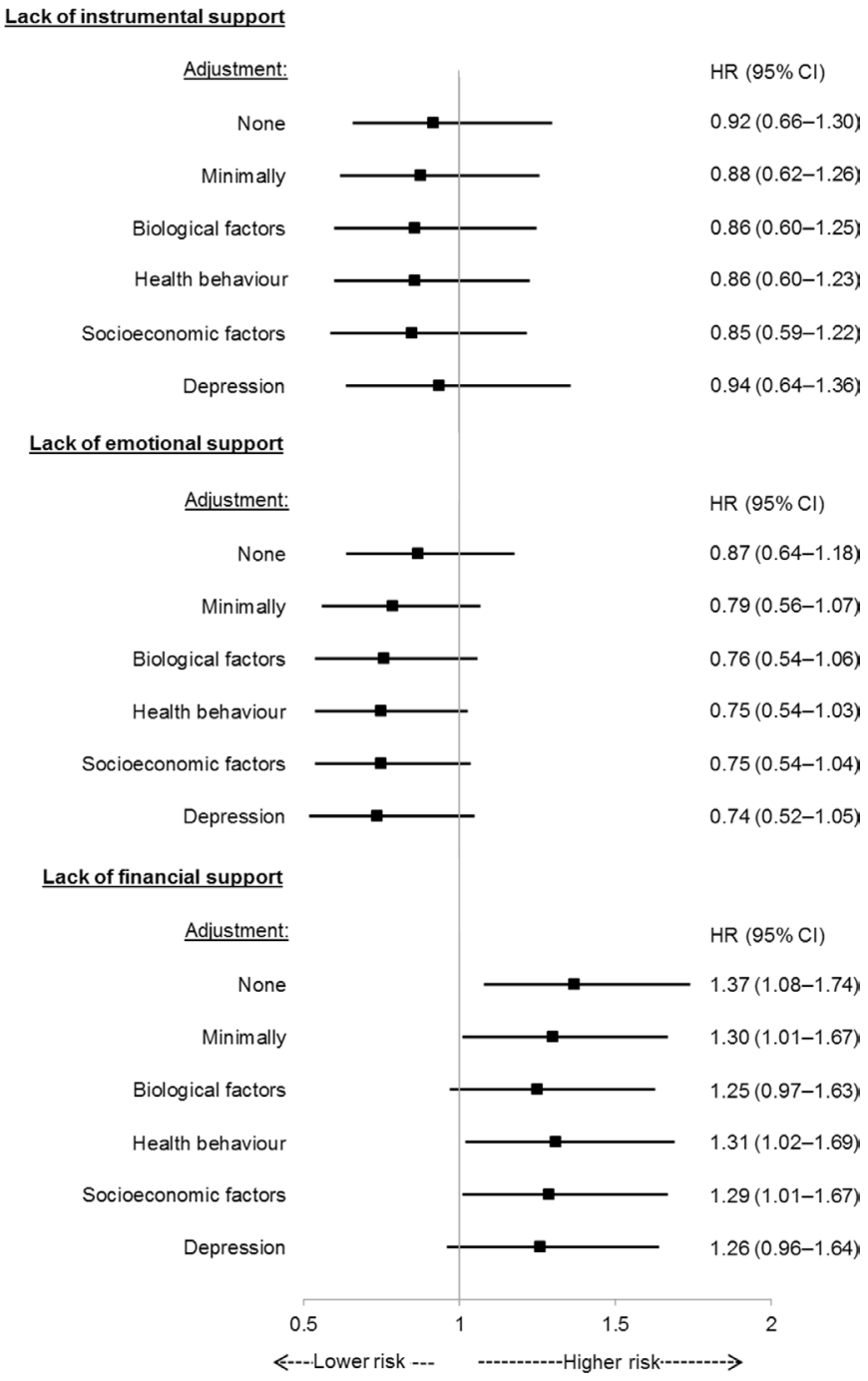

Figure 1 Association of different types of social support with incident cardiovascular events.

Participants with lack of social integration similar to participants with lack of instrumental support were more likely to be female, unemployed, physically inactive, had higher depression scores, lower income, a lower level of education and additionally were more often smokers.

\section{Association of social support at baseline with incident cardiovascular events and all-cause mortality during follow- up}

During the 13.4-year follow-up, 339 cardiovascular events (122 strokes, 183 coronary events, 34 deaths related to diseases of the circulatory system) and 530 deaths (98 cardiovascular, 432 other causes) occurred. Lack of financial support was associated with an increased cardiovascular event risk (minimally adjusted hazards ratio $=1.30$ (95\% CI 1.01 to 1.67$)$ ), which was partly explained by biological factors (1.25 (95\% CI 0.97 to 1.63$))$ and depression (1.26 (95\% CI 0.96 to 1.64$)$ ). Even though lack of financial support was significantly associated with both depression and low socioeconomic status, lack of financial support still predicted incident cardiovascular events in a multivariable model adjusted for socioeconomic factors (1.29 (95\% CI 1.01 to 1.67), figure 1). The association of instrumental and emotional support with cardiovascular event incidence was less strong (figure 1). None of the types of social support was consistently associated with all-cause mortality in adjusted models (figure 2).

\section{Association of social integration at baseline with incident cardiovascular events and all-cause mortality during follow- up}

Lack of social integration was associated with incident cardiovascular events (minimally adjusted HR 1.44 (95\% CI 0.97 to 2.14), figure 3 ), and with deaths due to all causes (minimally adjusted HR 1.47 (1.09-1.97), figure 4).

\section{Subgroup analyses stratified by age and sex}

Sex-stratified and age-stratified analyses showed that the association between lack of financial support and increased cardiovascular event incidence was stronger for men (minimally adjusted HR 1.54 (95\% CI 1.14 to 2.07)) than for women (0.89 (95\% CI 0.55 to 1.42 ), supplementary eTable 1 ) and stronger for younger ( $<65$ years, 1.53 (95\% CI 1.08 to 2.16$)$ ) than older ( $\geq 65$ years, 1.12 (95\% CI 0.78 to 1.60 ), supplementary eTable 2) participants. The association between lack of social integration and cardiovascular events was also stronger for men $(1.92$ (95\% CI 1.07 to 3.46$))$ than for women (1.12 (95\% CI 0.66 to 1.90$)$, supplementary eTable 1 ) and stronger for younger ( $<65$ years, 1.79 (95\% CI 1.01 to 3.18$)$ ) than older ( $\geq 65$ years, $1.10(95 \%$ CI 0.64 to 1.91 ), supplementary eTable 2) participants. Similarly, the association between lack of social integration and allcause mortality was also stronger for men $(2.45$ (95\% CI 1.54 to 3.87)) than for women (1.11 (95\% CI 0.77 to 1.62), supplementary eTable 3$)$ and stronger in younger (1.87 (95\% CI 1.22 to $2.88)$ ) than in older ( 1.17 (95\% CI 0.78 to 1.74$)$, supplementary eTable 4) participants.

\section{DISCUSSION \\ Principal findings}

In the population-based Heinz Nixdorf Recall study, which uses state-of-the-art population enrolment, assessment and endpoint evaluation methods, we for the first time demonstrate that lack of financial support was associated with a higher incidence of cardiovascular events, even when adjusted for cardiovascular risk factors, socioeconomic factors, health behaviour or emotional factors. When adding biological factors such as blood pressure, blood lipids and blood glucose as well as medications to control these factors or emotional factors, such as depression and medications to control depression to the model including age, sex and social integration, effect sizes of financial support were only slightly reduced (both by 4\%). In contrast to financial support, the association of instrumental and emotional support with cardiovascular event incidence was less strong, and none of the types of social support was consistently associated with all-cause mortality. Lack of social integration however was associated with both incident cardiovascular events and all-cause mortality.

\section{Comparison with other studies}

So far, only one study analysed the association of social support with a combined endpoint of incident cardiovascular disease, which was however based on a broader definition without validation by an endpoint committee as in our study. ${ }^{11}$ This analysis of data from the English Longitudinal Study of Ageing revealed that, similar to our results, the quality aspect of social relationships, operationalised as feeling lonely, predicted incident cardiovascular disease. In contrast to our study, the quantity 


\section{All-cause mortality}

Social support

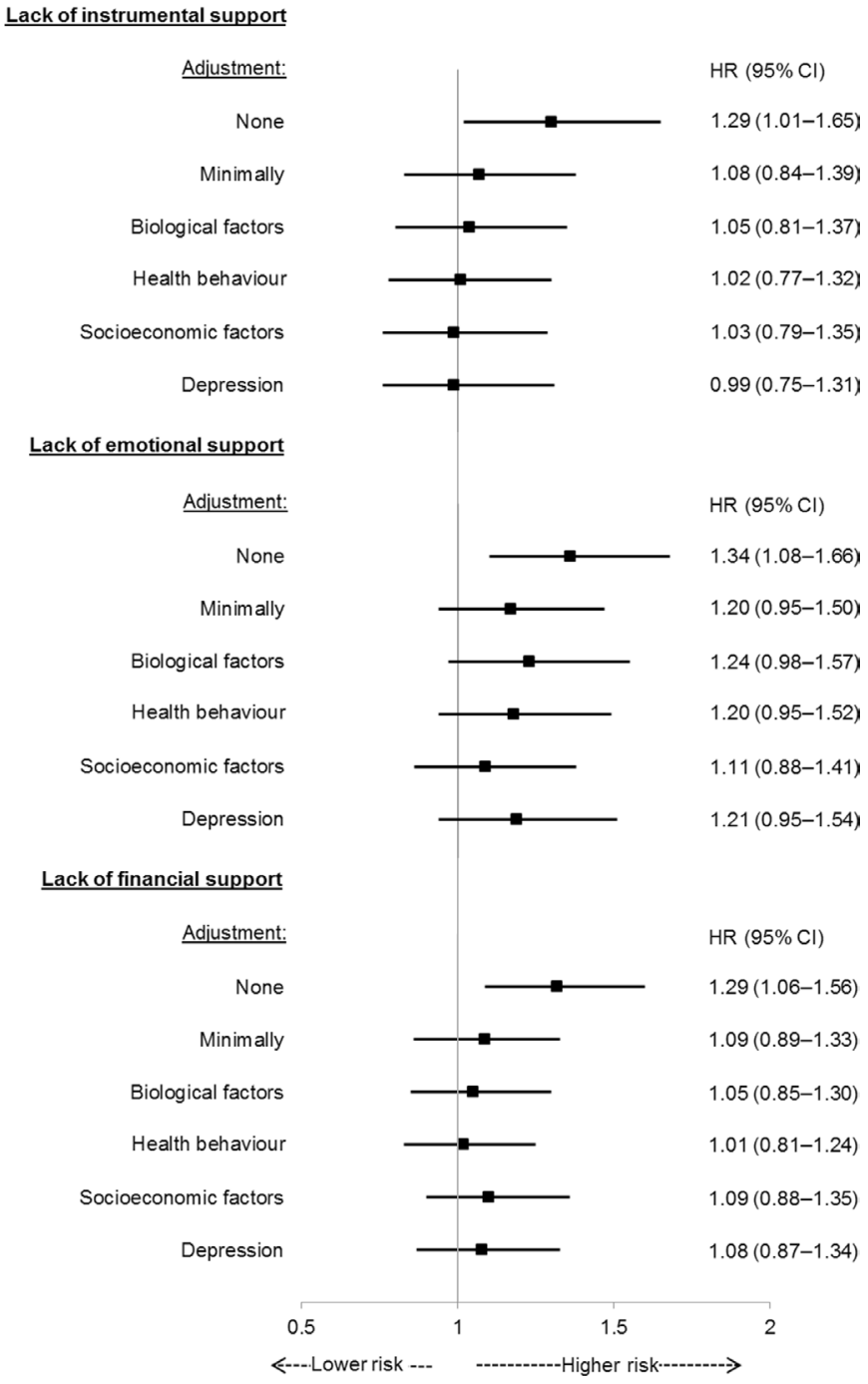

Figure 2 Association of different types of social support with allcause mortality.

\section{Cardiovascular events}

Social integration

Lack of social integration

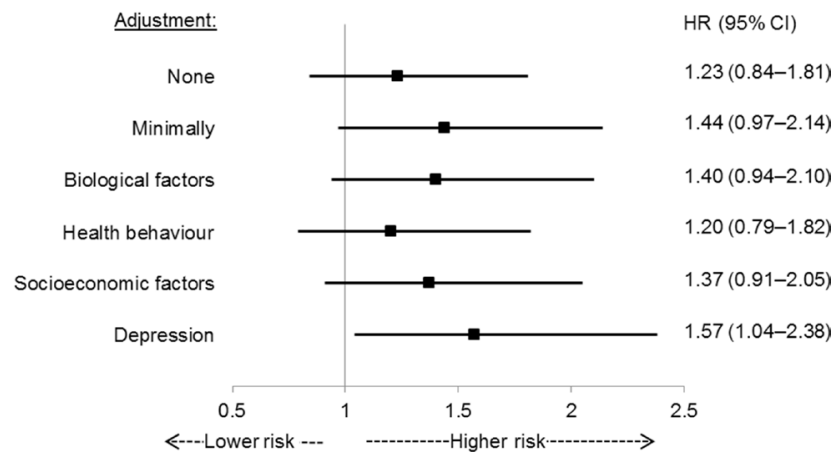

Figure 3 Association of social integration with incident cardiovascular events.

\section{All-cause mortality}

Social integration

Lack of social integration

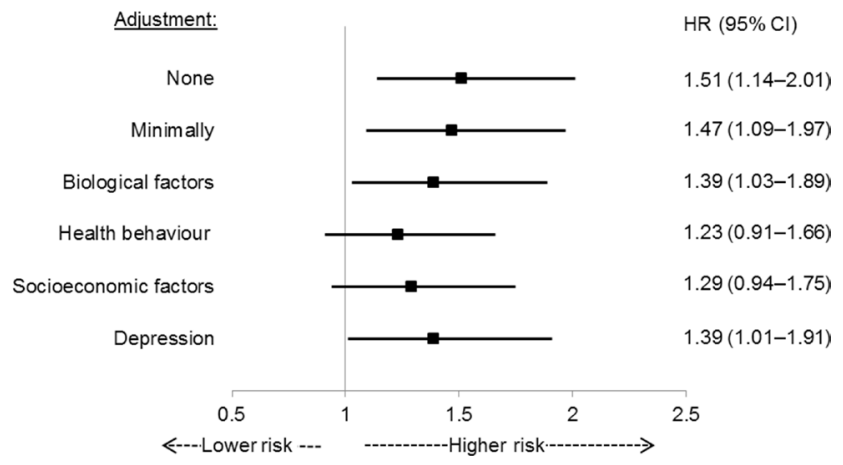

Figure 4 Association of social integration with all-cause mortality.

aspect, also operationalised by the concept of social isolation, did not predict incident cardiovascular disease. In contrast to our study, it cannot be disentangled, which types of social support participants were missing. Multivariable regression models were adjusted for sociodemographic and cardiovascular risk factors but not emotional factors as in our study. Depression, which is associated with increased cardiovascular risk by itself, ${ }^{12}$ can impair social contacts due to disturbed social behaviour. The role of depression in the association of loneliness and social isolation with incident stroke and myocardial infarction was supported by evidence of the population-based UK biobank cohort study. ${ }^{3}$ Similar to our results, participants of the UK biobank study, who reported loneliness and social isolation, were more likely to suffer from depressed mood, were more often smokers and had lower education and lower household income compared with those not reporting social isolation and loneliness. The authors also analysed the extent to which baseline biological, behavioural and socioeconomic factors as well as depression explained the association of loneliness and social isolation with stroke and myocardial infarction. Whereas the effect of social isolation was, similar to our results, only slightly explained by depression (8\% for acute myocardial infarction, $6 \%$ for incident stroke), the effect of loneliness was, in contrast to our results for social support, to a considerable degree explained by depression (20\% for myocardial infarction, $15 \%$ for stroke). These results highlight the importance of a clear definition of the different aspects of social relationships. ${ }^{8}$

We for the first time analysed prevalence and health consequences of three important types of support that is available via social relationships, that is, instrumental support, implying help in activities of daily living like buying groceries, house cleaning and preparing meals; emotional support, implying counselling and comforting in case of problems; and financial support, implying paying bills and household expenses. Our observation of an increased incidence of cardiovascular events when individuals feel that they have no financial support or did not get enough financial support might be explained by the stress response caused by this perception of lack of financial support. ${ }^{13}{ }^{14}$ Consistent with our results regarding lack of financial support, participants of the Jackson Heart study experiencing high financial stress were more likely to be depressed, were more often smokers and had lower education and income compared with participants not experiencing financial stress. ${ }^{14}$ In contrast to our results, which showed that the association of 
lack of financial support with incident cardiovascular disease was only slightly explained by depression (4\%), the Jackson Heart study showed the association of financial stress with coronary heart disease was considerably influenced by depression $(20 \%)$, which was also assessed with the Center for Epidemiological Studies - Depression Scale (CES-D), as in our study. Similar to our study, effect estimates were not considerably reduced after adjustment for socioeconomic, health behaviour and cardiovascular risk factors. ${ }^{14}$ Thus, it can be concluded that the association between perceived lack of financial support and elevated cardiovascular event risk is not solely based on increased cardiovascular risk factor burden, low socioeconomic status, unhealthy lifestyle or emotional problems. This supports the main effect model of social support, stating that social support has an overall beneficial effect in contrast to the buffering model stating that social support protects from potentially adverse effects of stressful situations. ${ }^{15}$

Contrary to financial support, instrumental and emotional support were not associated with incident cardiovascular events in our study. Especially for instrumental support, this may be caused by our method of assessing social support, since we only assessed the harmful effect of 'no' or 'not enough' support, but not of 'too much' support. Overprotective social support has been shown to increase cardiovascular reactivity in stressful situations due to threatening self-efficacy and autonomy. ${ }^{16} 17$ Furthermore, we did not analyse the effect of 'no support' and 'not enough' support separately due to lack of statistical power. Also, the quality of the relationship towards the supporting persons might be relevant, since receiving support from ambivalent persons, who sometimes provide and sometimes do not provide support, has been shown to increase stress responses. ${ }^{18}$ Additionally, receiving high amounts of support can cause feelings of commitment to return the support received due to the reciprocal nature of social relationships. ${ }^{19}$ Unfortunately, only reception, but not provision of social support, was assessed in the present study.

Regarding the outcome of all-cause mortality, previous studies similar to our observation revealed that quality aspects of social relationships, like feeling supported, were not predictive. ${ }^{20}$ All-cause mortality was rather predicted by quantitative aspects of social relationships, like social network size, diversity or frequency of contact. ${ }^{20} 21$ This evidence suggests that quantitative aspects of social relationships may be more important to support recovery after illness than prevent incidence of new disease. Support for this theory comes from the UK biobank study, which showed that social isolation, but not loneliness, was associated with increased mortality in participants with a history of stroke or myocardial infarction. ${ }^{3}$

The influence of age and sex on the association between different aspects of social relationships and incident cardiovascular events as well as all-cause mortality has not been systematically analysed before. We for the first time observed that the association of financial support and social integration with incident cardiovascular events was stronger for men than for women and stronger for younger than older participants. The association between social integration and all-cause mortality was also stronger for men than for women supporting results of the British Whitehall II cohort study, which showed that a low level of social contacts was significantly associated with increased allcause and cardiovascular mortality in men but not in women. ${ }^{20}$ We observed a stronger association between social integration and all-cause mortality in younger than in older participants contradicting previous results from a smaller American healthcare cohort with a broader age range, which revealed that social network measures were significantly associated with all-cause mortality only in persons $\geq 75$ years. $^{21}$

\section{Clinical implications}

Although scientific evidence confirms that social relationships are a major determinant of cardiovascular morbidity and mortality with effects being as strong as or even exceeding that of wellestablished clinical risk factors such as blood pressure, cholesterol and obesity, ${ }^{42}$ they are often ignored in clinical practice. To improve awareness of social factors in healthcare, the American Centers for Medicare and Medicaid Services are currently testing whether systematically identifying and addressing social health-related risk factors can actually improve individual and population health and reduce healthcare costs. ${ }^{23} 24$ The causal mechanisms through which social relationships affect health remain to be explored to be able to customise targeted interventions. ${ }^{22}$

\section{Key messages}

What is already known on this subject?

- Social relationships have been associated with cardiovascular morbidity, cardiovascular mortality and all-cause mortality.

\section{What might this study add?}

- Previous studies did not further characterise different aspects of social relationships. In the population-based prospective Heinz Nixdorf Recall study, we analysed how both qualitative aspects of social relationships (perceived adequacy of instrumental, emotional and financial support from social relationships) and quantitative aspects of social relationships (number of social contacts, social integration) were associated with incident cardiovascular events and allcause mortality. Using state-of-the-art population enrolment, assessment and endpoint evaluation techniques, we showed that especially lack of financial support was associated with increased cardiovascular event incidence, whereas lack of social integration was associated with increased all-cause mortality.

\section{How might this impact on clinical practice? \\ - Qualitative and quantitative aspects of social relationships should better be captured and targeted in cardiovascular diseases.}

Acknowledgements The authors would like to express their gratitude to all study participants of the Heinz Nixdorf Recall (HNR) Study, the personnel of the HNR study centre and the EBT-scanner facilities, the investigative group and all former employees of the HNR study. The authors would also like to thank the Advisory Board of the HNR study: T Meinertz, Hamburg, Germany (Chair); C Bode, Freiburg, Germany; P J de Feyter, Rotterdam, Netherlands; B Güntert, Hall i.T., Austria; F Gutzwiller, Bern, Switzerland; H Heinen, Bonn, Germany; 0 Hess ( $\dagger$ ), Bern, Switzerland; B Klein ( $\dagger$ ), Essen, Germany; H Löwel, Neuherberg, Germany; M Reiser, Munich, Germany; G Schmidt (†), Essen, Germany; M Schwaiger, Munich, Germany; C Steinmüller, Bonn, Germany; T Theorell, Stockholm, Sweden; and S N Willich, Berlin, Germany. Criteria and endpoint committee: C Bode, Freiburg, Germany (Chair); K Berger, Münster, Germany; M Dichgans, München, Germany; H R Figulla, Jena, Germany; C Hamm, Bad Nauheim, Germany; P Hanrath, Aachen, Germany; W Köpcke, Münster, Germany; B Ringelstein, Münster, Germany; C Weimar, Essen, Germany; and A Zeiher, Frankfurt, Germany.

Contributors Conception or design of the work: RE, K-HJ and JS. Data collection: BS and SW. Data analysis: JG and RK. Data interpretation: all authors. Drafting the article: JG. Critical revision of the article: all authors. Final approval of the version to be published: all authors. 
Funding The authors would like to thank the Heinz Nixdorf Foundation (chairman: Martin Nixdorf; past chairman: Dr jur. Gerhard Schmidt ( $(t))$, for their generous support of this study. Parts of the study were also supported by the German Research Council (DFG) (DFG project: El 969/2-3, ER 155/6-1;6-2, HO 3314/21;2-2;2-3;4-3, INST 58219/32-1, J0 170/8-1, KN 885/3-1, PE 2309/2-1, SI 236/8-1;9-1;10-1), the German Ministry of Education and Science (BMBF project: 01EG0401, 01GI0856, 01GI0860, 01GS0820_WB2-C, 01ER1001D, 01GI0205), the Ministry of Innovation, Science, Research and Technology, North Rhine-Westphalia (MIWFT-NRW), the Else Kröner-Fresenius-Stiftung (project: 2015_A119) and the German Social Accident Insurance (DGUV project: FF-FP295). Furthermore, the study was supported by the Competence Network for HIVIAIDS, the deanship of the University Hospital and IFORES of the University Duisburg-Essen, the European Union, the German Competence Network Heart Failure, Kulturstiftung Essen, the Protein Research Unit within Europe (PURE), the Dr. Werner-Jackstädt Stiftung and the following companies: Celgene GmbH München, Imatron/GE-Imatron, Janssen, Merck KG, Philips, ResMed Foundation, Roche Diagnostics, Sarstedt AG\&Co, Siemens HealthCare Diagnostics and Volkswagen Foundation.

\section{Competing interests None declared.}

Patient and public involvement Patients and/or the public were not involved in the design, or conduct, or reporting, or dissemination plans of this research.

Patient consent for publication Not required.

Provenance and peer review Not commissioned; externally peer reviewed.

Data availability statement Data are available on reasonable request. The corresponding author has full access to all data in the study and final responsibility for the submission of the article for publication. Due to data security reasons (ie, data contain potentially participant identifying information), the HNR study does not allow sharing data as a public use file. Data requests can also be addressed to: recall@uk-essen.de.

Open access This is an open access article distributed in accordance with the Creative Commons Attribution Non Commercial (CC BY-NC 4.0) license, which permits others to distribute, remix, adapt, build upon this work non-commercially, and license their derivative works on different terms, provided the original work is properly cited, appropriate credit is given, any changes made indicated, and the use is non-commercial. See: http://creativecommons.org/licenses/by-nc/4.0/.

ORCID iD

Janine Gronewold http://orcid.org/0000-0002-1470-424X

\section{REFERENCES}

1 Valtorta NK, Kanaan M, Gilbody S, et al. Loneliness and social isolation as risk factors for coronary heart disease and stroke: systematic review and meta-analysis of longitudinal observational studies. Heart 2016;102:1009-16.

2 Holt-Lunstad J, Smith TB, Layton JB, et al. Social relationships and mortality risk: a meta-analytic review. PLoS Med 2010;7:e1000316.

3 Hakulinen C, Pulkki-Råback L, Virtanen M, et al. Social isolation and loneliness as risk factors for myocardial infarction, stroke and mortality: UK Biobank cohort study of 479054 men and women. Heart 2018;104:1536-42.

4 Pantell M, Rehkopf D, Jutte $D$, et al. Social isolation: a predictor of mortality comparable to traditional clinical risk factors. Am J Public Health 2013;103:2056-62.
5 Thoits PA. Mechanisms linking social ties and support to physical and mental health. J Health Soc Behav 2011;52:145-61.

6 Liao CC, Yeh CJ, Lee SH, et al. Providing instrumental social support is more beneficial to reduce mortality risk among the elderly with low educational level in Taiwan: a 12-year follow-up national longitudinal study. J Nutr Health Aging 2015;19:447-53.

7 Schmermund A, Möhlenkamp S, Stang A, et al. Assessment of clinically silent atherosclerotic disease and established and novel risk factors for predicting myocardial infarction and cardiac death in healthy middle-aged subjects: rationale and design of the Heinz Nixdorf recall study. risk factors, evaluation of coronary calcium and lifestyle. Am Heart J 2002;144:212-8.

8 Seeman TE, Berkman LF. Structural characteristics of social networks and their relationship with social support in the elderly: who provides support. Soc Sci Med 1988;26:737-49.

9 Weyers S, Dragano N, Möbus S, et al. Low socio-economic position is associated with poor social networks and social support: results from the Heinz Nixdorf recall study. Int J Equity Health 2008;7:13.

10 Berkman LF, Melchior M, Chastang J-F, et al. Social integration and mortality: a prospective study of French employees of electricity of France-Gas of France: the GAZEL cohort. Am J Epidemiol 2004:159:167-74.

11 Valtorta NK, Kanaan M, Gilbody S, et al. Loneliness, social isolation and risk of cardiovascular disease in the English longitudinal study of ageing. Eur J Prev Cardiol 2018;25:1387-96.

12 Van der Kooy K, van Hout H, Marwijk H, et al. Depression and the risk for cardiovascular diseases: systematic review and meta analysis. Int $J$ Geriatr Psychiatry 2007;22:613-26

13 Drentea P. Age, debt and anxiety. J Health Soc Behav 2000;41:437-50.

14 Moran KE, Ommerborn MJ, Blackshear CT, et al. Financial stress and risk of coronary heart disease in the Jackson heart study. Am J Prev Med 2019;56:224-31.

15 Cohen S, Wills TA, Stress WTA. Stress, social support, and the buffering hypothesis. Psychol Bull 1985;98:310-57.

16 Berkhuysen MA, Nieuwland W, Buunk BP, et al. Change in self-efficacy during cardiac rehabilitation and the role of perceived overprotectiveness. Patient Educ Couns 1999;38:21-32.

17 Zniva R, Pauli P, Schulz SM. Overprotective social support leads to increased cardiovascular and subjective stress reactivity. Biol Psychol 2017;123:226-34.

18 Holt-Lunstad J, Clark BD. Social stressors and cardiovascular response: influence of ambivalent relationships and behavioral ambivalence. Int J Psychophysiol 2014:93:381-9.

19 Buunk BP, Hoorens V. Social support and stress: the role of social comparison and social exchange processes. Br J Clin Psychol 1992;31:445-57.

20 Stringhini S, Berkman L, Dugravot A, et al. Socioeconomic status, structural and functional measures of social support, and mortality: the British Whitehall II cohort study, 1985-2009. Am J Epidemiol 2012;175:1275-83.

21 Vogt TM, Mullooly JP, Ernst D, et al. Social networks as predictors of ischemic heart disease, cancer, stroke and hypertension: incidence, survival and mortality. J Clin Epidemiol 1992;45:659-66.

22 House J, Landis K, Umberson D. Social relationships and health. Science 1988;241:540-5.

23 Adler NE, Stead WW. Patients in context--EHR capture of social and behavioral determinants of health. N Engl J Med 2015;372:698-701.

24 Alley DE, Asomugha CN, Conway PH, et al. Accountable Health Communities-Addressing Social Needs through Medicare and Medicaid. N Engl J Med 2016;374:8-11. 